\title{
An Analytical Model for Adsorption and Diffusion of Atoms/Ions on Graphene Surface
}

\author{
Yan-Zi Yu, Jian-Gang Guo, and Yi-Lan Kang \\ Tianjin Key Laboratory of Modern Engineering Mechanics, School of Mechanical Engineering, Tianjin University, \\ Tianjin 300072, China \\ Correspondence should be addressed to Jian-Gang Guo; guojg@tju.edu.cn
}

Received 24 July 2015; Accepted 16 September 2015

Academic Editor: Ungyu Paik

Copyright (C) 2015 Yan-Zi Yu et al. This is an open access article distributed under the Creative Commons Attribution License, which permits unrestricted use, distribution, and reproduction in any medium, provided the original work is properly cited.

\begin{abstract}
Theoretical investigations are made on adsorption and diffusion of atoms/ions on graphene surface based on an analytical continuous model. An atom/ion interacts with every carbon atom of graphene through a pairwise potential which can be approximated by the Lennard-Jones (L-J) potential. Using the Fourier expansion of the interaction potential, the total interaction energy between the adsorption atom/ion and a monolayer graphene is derived. The energy-distance relationships in the normal and lateral directions for varied atoms/ions, including gold atom $(\mathrm{Au})$, platinum atom $(\mathrm{Pt})$, manganese ion $\left(\mathrm{Mn}^{2+}\right)$, sodium ion $\left(\mathrm{Na}^{1+}\right)$, and lithium-ion $\left(\mathrm{Li}^{1+}\right)$, on monolayer graphene surface are analyzed. The equilibrium position and binding energy of the atoms/ions at three particular adsorption sites (hollow, bridge, and top) are calculated, and the adsorption stability is discussed. The results show that $\mathrm{H}$-site is the most stable adsorption site, which is in agreement with the results of other literatures. What is more, the periodic interaction energy and interaction forces of lithium-ion diffusing along specific paths on graphene surface are also obtained and analyzed. The minimum energy barrier for diffusion is calculated. The possible applications of present study include drug delivery system (DDS), atomic scale friction, rechargeable lithium-ion graphene battery, and energy storage in carbon materials.
\end{abstract}

\section{Introduction}

In recent decade, graphene has been paid much attention as an ideal candidate for the development of high-sensitivity sensors [1-3], drug delivery system (DDS) [4-7], rechargeable lithium-ion graphene battery [8-10], lithium storage in carbon materials [11-15], and so on, due to its unique characteristics, such as the thinnest known 2-dimensional nanomaterial, the lowest resistivity, and extreme mechanical strength. The characteristics of graphene have been widely investigated in the field of biology, chemistry, and physics. However, there is still a common unsolved major scientific question for its applications. It is to develop an analytical model to quantitatively describe the adsorption and diffusion of atoms/ions on graphene surface, which can be further applied for the interaction between nanoparticles or atomic force microscopy (AFM) tip and graphene surface.

For this problem, lots of theoretical and experimental researches have been made, and some interesting research results are achieved. In atomic and nanoscale, general theoretical research methods include density functional theory (DFT), first principle, and molecular dynamics (MD) simulation. A systematic density functional study was performed on the adsorption of $\mathrm{Cu}, \mathrm{Ag}$, and $\mathrm{Au}$ adatoms on pristine graphene, especially accounting for van der Waals (vdW) interactions by the vdW-DFT and Perdew, Burke, and Ernzerhof plus long-range dispersion correction (PBE + D2) methods [16]. According to the differences of the total energies at the three adsorption sites, they found that the diffusion of $\mathrm{Cu}$ and $\mathrm{Au}$ took place along carboncarbon bonds, while the Ag adatoms could diffuse almost unrestrictedly on graphene sheet. Using density functional theory, theoretical calculations were also carried out for the diffusion of $\mathrm{Si}$ and $\mathrm{C}$ atoms on a monolayer graphene surface and between the multilayer graphene layers [17]. The results showed that the diffusion barrier for a $\mathrm{Si}$ atom on monolayer graphene surface was extremely small, and the diffusion of Si atom between bilayer graphene was also almost 
barrier-free. However, the diffusion of a $\mathrm{C}$ atom on monolayer graphene was far more difficult than that of the Si atom. By the same methods, the adsorption of $\mathrm{Li}$ atom on graphene was investigated [9]. Three different adsorption sites were taken into consideration. According to their researches, the hollow site was found to be the most stable, followed by the bridge and top sites. Moreover for $\mathrm{Li}$ atom, the minimum diffusion energy path where $\mathrm{Li}$ atom migrates from a hollow site to a neighboring hollow site should be a path through the bridge site between them. What is more, the adsorption, diffusion, and desorption of hydrogen on graphene were studied by DFT method [18]. They concluded that the atomic hydrogen adsorbate produced by bombarding the surface with an atomic beam was not possible under equilibrium conditions, and below room temperature diffusion was frozen out and a beam-deposited atomic hydrogen adsorbate could be maintained, though completely out of equilibrium.

Apart from density function theory method, MD simulation methods have also been widely used in the study of graphene interacting with atom/ion. The diffusion processes of the lithium-ion on a fluorinated graphene (F-graphene) surface were investigated by means of a direct molecular orbital-molecular dynamics (MO-MD) method [19]. It was found that the diffusion coefficient of lithium-ion on Fgraphene surface was close to that of normal graphene $(\mathrm{H}-$ graphene), but the thermal behavior on the F-graphene surface was much different from that on the $\mathrm{H}$-graphene surface. The direction of lithium-ion diffusion could be controlled by fluorinated substitution (F-substitution) on the graphene sheet around room temperature. But at higher temperatures, the lithium-ion diffused freely on surface and edge region of $\mathrm{F}$-graphene. The interaction of $\mathrm{Mg}$ atom with graphene was also researched by using MD simulations [20]. It was found that the $\mathrm{Mg}$ atom vibrated strongly on the graphene surface; even at high temperature $(1000 \mathrm{~K})$, the diffusion of $\mathrm{Mg}$ is difficult.

There are relatively few experimental researches on adsorption and diffusion of atoms/ions on graphene surface. The viability of utilizing functionalized graphene oxide with good solubility and stability, biocompatibility, and targetspecificity was demonstrated as a drug nanocarrier for controlled loading and folate receptor-targeted drug delivery of anticancer drugs [21]. It revealed that mastering the stability of equilibrium for graphene was vital to investigate a new drug delivery system for cancer therapy. The possibility of higher lithium storage capacity was explored by controlling layered structures of graphene nanosheet materials [12]. It enlightened that the spacing equilibrium distance of atom/ion on and between graphene sheets is critical for the future large-scale, high-capacity lithium-ion batteries. The diffusion of a cobalt bis-terpyridine, $\mathrm{Co}(\mathrm{tpy})_{2}$-containing tripodal compound $(1 \bullet 2 \mathrm{PF} 6)$, noncovalently adsorbed on the surface of a single-layer graphene (SLG), was studied by scanning electrochemical microscopy [22]. It was proved that surface diffusion is the main mechanism for the observed decrease in the electrochemical response due to radial diffusion of the adsorbed molecules outward from the microspots onto the unfunctionalized areas of the SLG surface.
In order to quantitatively understand atomic adsorption and diffusion on graphene surface from the perspective of atomic scale, an analytical continuous model is put forward in this work based on the Fourier expansion and Lennard-Jones potential. With the theoretical model, equilibrium position, binding energy, and adsorption stability of the atoms/ions $\left(\mathrm{Au}, \mathrm{Pt}, \mathrm{Mn}^{2+}, \mathrm{Na}^{1+}\right.$, and $\mathrm{Li}^{1+}$ ) on monolayer graphene surface are calculated and analyzed. The different metal atoms/ions selected in this work possess many application prospects. For example, $\mathrm{Mn}^{2+}$ is an ideal candidate for drug delivery and $\mathrm{Li}^{1+}$ is a common element for high-energy density rechargeable alkali batteries. The dependence of diffusion energy and diffusion forces on the path is illustrated.

\section{Theoretical Model}

A monolayer graphene is assumed to be perfect, flat, and infinite compared to atomic scale (as shown in Figure 1(a)). Due to its periodic honeycomb lattice structure, a twodimensional lattice vector parallel to graphene can be defined [23-25]:

$$
\mathbf{l}=l_{1} \mathbf{a}_{\mathbf{1}}+l_{2} \mathbf{a}_{2}
$$

where $l_{1}$ and $l_{2}$ are integers and $\mathbf{a}_{1}$ and $\mathbf{a}_{2}$ are the unit lattice vectors in the plane (as shown in Figure 1(a)). It is evident to know that the translation of a single adsorption atom by 1 will take it from one position above a surface lattice cell into an equivalent position above another different lattice cell (as shown in Figure 1(a)). Therefore, the total interaction potential $u(\mathbf{r})$ takes the form of

$$
u(\mathbf{r}+\mathbf{l})=u(\mathbf{r}),
$$

where $\mathbf{r}$ is the position of the adsorption atom with respect to the graphene surface.

Thus, the periodic nature of the total interaction potential $u(\mathbf{r})$ is a Fourier series:

$$
u(\mathbf{r})=\sum_{g} w_{g}(z) \exp (i \mathbf{g} \cdot \boldsymbol{\tau}),
$$

where $z$ is perpendicular distance between the adsorption atom and graphene surface, $\boldsymbol{\tau}$ is the two-dimensional translation vector, and $\mathbf{g}$ is the multiples of the reciprocal lattice vectors $\mathbf{b}_{1}$ and $\mathbf{b}_{2}$ :

$$
\mathbf{g}=2 \pi\left(g_{1} \mathbf{b}_{1}+g_{2} \mathbf{b}_{2}\right)
$$

where $g_{1}$ and $g_{2}$ are integers and $\mathbf{b}_{\mathbf{1}}$ and $\mathbf{b}_{\mathbf{2}}$ are defined by

$$
\begin{aligned}
& \mathbf{a}_{1} \cdot \mathbf{b}_{1}=\mathbf{a}_{2} \cdot \mathbf{b}_{2}=1, \\
& \mathbf{a}_{1} \cdot \mathbf{b}_{2}=\mathbf{a}_{2} \cdot \mathbf{b}_{1}=0 .
\end{aligned}
$$

Equation (5) indicates that $\mathbf{b}_{1}$ and $\mathbf{b}_{2}$ are perpendicular to $\mathbf{a}_{2}$, $\mathbf{a}_{1}$ and of length $\csc \varphi / a_{1}, \csc \varphi / a_{2}$, respectively, where $\varphi$ is 


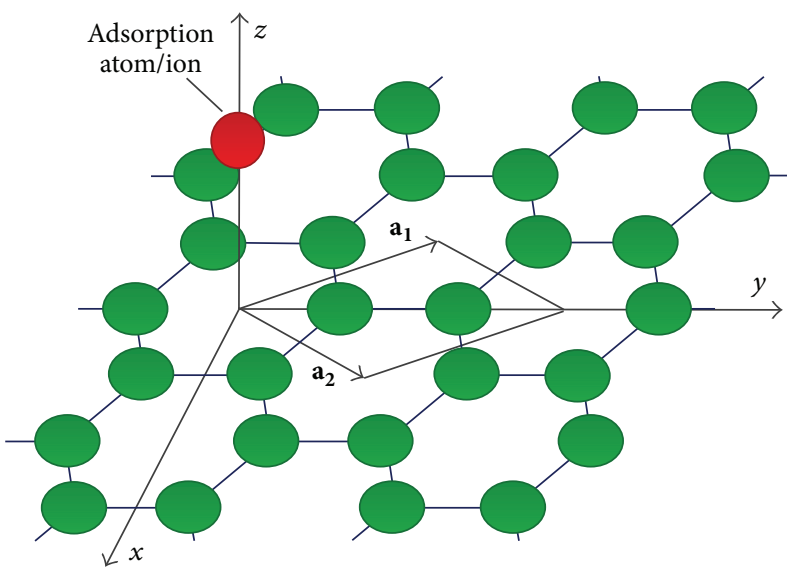

(a)

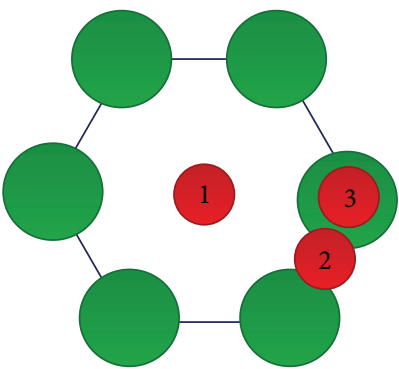

(1) H-site

(2) B-site

(3) T-site

(b)

FIGURE 1: Schematics of the graphene where (a) the rhombus connecting the centers of four adjacent hexagonal is selected as a twodimensional primitive unit cell; (b) hexagon with three particular adsorption sites: hollow (on top of a hexagon, $\mathrm{H}-$ ), top (on top of a C-C bond, T-), and bridge (on top of a carbon atom, B-).

the angle between $\mathbf{a}_{\mathbf{1}}$ and $\mathbf{b}_{\mathbf{1}} \cdot w_{g}(z)$ in (3) can be expressed in the following forms:

$$
\begin{gathered}
w_{g}(z)=\frac{1}{A} \sum_{l_{1}, l_{2}} \sum_{k} \int_{A} \exp (-i \mathbf{g} \cdot \boldsymbol{\tau}) u_{s}\left(z, \boldsymbol{\tau}+\mathbf{1}+\mathbf{m}_{k}\right) d \boldsymbol{\tau} \\
=\frac{1}{A} \sum_{k} \exp \left(i \mathbf{g} \cdot \mathbf{m}_{k}\right) \int_{0}^{\infty} \exp (-\mathrm{ig} \cdot \mathbf{t}) u_{s}(z, \mathbf{t}) d \mathbf{t}
\end{gathered}
$$

where $A$ is the area of unit cell, $u_{s}\left(z, \boldsymbol{\tau}+\mathbf{l}+\mathbf{m}_{k}\right)$ is the partial interaction potential between the single atom and the carbon atom in graphene plane, $k$ indicates the $k$ th atom in a unit cell, and the position of the atom in the plane is given by $\mathbf{l}+\mathbf{m}_{k}$ and $\mathbf{t}=\boldsymbol{\tau}+\mathbf{l}+\mathbf{m}_{k}$.

Commonly, the average interaction potential per atom/ion on graphene is defined by the total energy of an atom/ion, a sheet of graphene, and the graphene with atom/ion adsorption, which consider the effects of temperature, chemical bond, charge transfer, van der Waals interaction, and so forth $[9,10,26,27]$. In this paper, for the convenience of discussion, the interaction potential is only defined by van der Waals interaction. Even so, the model in this paper is also suitable for taking into account the effects of other factors mentioned above. Two empirical potentials commonly used are the L-J potential and the Morse potential. By referring the reader for detail of the Morse potential and its applications $[28,29]$, this paper adopts the form of 6-12 L-J potential to determine the van der Waals interaction potential between the adsorption atom and the $i$ th carbon atom in the graphene,

$$
u_{s}\left(\mathbf{r}_{i}\right)=4 \varepsilon\left[\left(\frac{\sigma}{r_{i}}\right)^{12}-\left(\frac{\sigma}{r_{i}}\right)^{6}\right]
$$

where $\mathbf{r}_{i}, \varepsilon$, and $\sigma$ denote the distance between the adsorption atom and the $i$ th carbon atom in the graphene, the potential well depth, and the distance when the interaction potential is equal to zero, respectively. Then,

$$
\begin{aligned}
& w_{0}(z)=\varepsilon \frac{2 \pi}{A} q\left(\frac{2 \sigma^{12}}{5 z^{10}}-\frac{\sigma^{6}}{z^{4}}\right), \\
& w_{g}(z) \\
& \quad=\varepsilon \frac{2 \pi}{A}\left[\frac{\sigma^{12}}{30}\left(\frac{g}{2 z}\right)^{5} K_{5}(g z)-2 \sigma^{6}\left(\frac{g}{2 z}\right)^{2} K_{2}(g z)\right],
\end{aligned}
$$

where $q=2$ is the number of the carbon atoms in the unit cell and $K_{2}$ and $K_{5}$ are the modified Bessel function of the second kind. Substitute (8) into (3), and then the total interaction potential $u(\mathbf{r})$ can be derived as

$$
\begin{aligned}
u(\mathbf{r}) & \approx w_{0}(z)+\sum_{g>0} \sum_{k=1}^{q} w_{g}(z) \exp \left[i \mathbf{g} \cdot\left(\mathbf{m}_{k}+\boldsymbol{\tau}\right)\right] \\
& =C(z)+\sum_{g>0} \sum_{k=1}^{q} D(g, z) \exp \left[i \mathbf{g} \cdot\left(\mathbf{m}_{k}+\boldsymbol{\tau}\right)\right],
\end{aligned}
$$

where $g$ is the magnitude of $\mathbf{g}$,

$$
\begin{aligned}
& g=|\mathbf{g}|=\frac{4 \pi}{\sqrt{3} a}\left(g_{1}^{2}+g_{2}^{2}-g_{1} g_{2}\right)^{1 / 2}, \\
& C(z)=w_{0}(z)=\varepsilon \frac{2 \pi}{A} q\left(\frac{2 \sigma^{12}}{5 z^{10}}-\frac{\sigma^{6}}{z^{4}}\right), \\
& D(g, z)=w_{g}(z) \\
& =\varepsilon \frac{2 \pi}{A}\left[\frac{\sigma^{12}}{30}\left(\frac{g}{2 z}\right)^{5} K_{5}(g z)-2 \sigma^{6}\left(\frac{g}{2 z}\right)^{2} K_{2}(g z)\right] .
\end{aligned}
$$

As shown in Figure 1(a), when $\boldsymbol{\tau}$ is chosen to be zero at a hollow point, and the unit cell which contains two carbon atoms is marked by a rhombus, then $\mathbf{m}_{k}$ for the two atoms in 
the unit cell indicated in Figure 1(a) are located at $(1 / 3,1 / 3) a$, $(2 / 3,2 / 3) a$, where $a$ is the distance between the centers of two nearest hexagons. Summation over $\mathbf{m}_{k}$ gives a value of $\cos \left[(2 \pi / 3)\left(g_{1}+g_{2}\right)\right]$, so $(9)$ can be further written as

$$
\begin{aligned}
& u(r) \\
& =C(z) \\
& \quad+2 \sum_{g>0} D(g, z) \exp (\mathbf{i g} \cdot \boldsymbol{\tau}) \cos \left[\frac{2 \pi}{3}\left(g_{1}+g_{2}\right)\right] .
\end{aligned}
$$

Because $D(g, z)$ in (11) decreases rapidly with the increase of $\boldsymbol{g}$, and the $\boldsymbol{\tau}$-dependent terms are important only over a short range of distance $z[23,25]$, there are approximately simplified expressions of $|\mathbf{g}|=4 \pi / \sqrt{3} a$ and $\cos \left[(2 \pi / 3)\left(g_{1}+\right.\right.$ $\left.\left.g_{2}\right)\right]=-1 / 2$. As shown in Figure 1(a), $x$ and $y$ coordinates are marked; $\boldsymbol{\tau}=\mathbf{x}+\mathbf{y}$ can be expressed in terms of $\mathbf{a}_{\mathbf{1}}$ and $\mathbf{a}_{\mathbf{2}}$ with

$$
\begin{aligned}
& \mathbf{x}=\frac{x}{a}\left(\mathbf{a}_{1}-\mathbf{a}_{2}\right), \\
& \mathbf{y}=\frac{y}{\sqrt{3}}\left(\mathbf{a}_{1}+\mathbf{a}_{2}\right) .
\end{aligned}
$$

Thus, the total energy between an adsorption atom/ion and graphene can be derived as

$$
\begin{aligned}
& u(r)=C(z)-D\left(\frac{4 \pi}{\sqrt{3} a}, z\right) \\
& \cdot\left[2 \cos \left(\frac{2 \pi y}{\sqrt{3} a}\right) \cos \left(\frac{2 \pi x}{a}\right)+\cos \left(\frac{4 \pi y}{\sqrt{3} a}\right)\right] .
\end{aligned}
$$

If we introduce the following dimensionless variables $A^{*}=$ $A / a^{2}, z^{*}=z / a, g^{*}=g a, \sigma^{*}=\sigma / a, x^{*}=x / a, y^{*}=y / a$, and $u^{*}=u / \varepsilon$, then the dimensionless form of total energy can be written as

$$
\begin{aligned}
u^{*}(r) & \lambda q\left(\frac{2 \sigma^{* 12}}{5 z^{* 10}}-\frac{\sigma^{* 6}}{z^{* 4}}\right)-\lambda(\alpha-\beta) \\
& \cdot\left[2 \cos \left(\frac{2 \pi}{\sqrt{3}} y^{*}\right) \cos \left(2 \pi x^{*}\right)+\cos \left(\frac{4 \pi}{\sqrt{3}} y^{*}\right)\right],
\end{aligned}
$$

where dimensionless parameters $\lambda, \alpha$, and $\beta$ have the following forms:

$$
\begin{aligned}
& \lambda=\frac{2 \pi}{A^{*}}, \\
& \alpha=\frac{\sigma^{* 12}}{30}\left(\frac{g^{*}}{2 z^{*}}\right)^{5} K_{5}\left(g^{*} z^{*}\right), \\
& \beta=2 \sigma^{* 6}\left(\frac{g^{*}}{2 z^{*}}\right)^{2} K_{2}\left(g^{*} z^{*}\right) .
\end{aligned}
$$

Furthermore, let $\mathbf{F}^{*}=-\nabla u^{*}\left(\mathbf{r}^{*}\right)$, and then the normal and lateral interaction forces between the atom/ion and graphene can be obtained in the Cartesian coordinate system as shown in Figure 1(a),

$$
\begin{aligned}
F_{x}^{*} & =-2 \pi \lambda(\alpha-\beta)\left[2 \cos \left(\frac{2 \pi}{\sqrt{3}} y^{*}\right) \sin \left(2 \pi x^{*}\right)\right], \\
F_{y}^{*} & =-\frac{4 \pi \lambda}{\sqrt{3}}(\alpha-\beta) \\
\cdot & {\left[\sin \left(\frac{2 \pi}{\sqrt{3}} y^{*}\right) \cos \left(2 \pi x^{*}\right)+\sin \left(\frac{4 \pi}{\sqrt{3}} y^{*}\right)\right], } \\
F_{z}^{*} & =4 \lambda q\left(\frac{\sigma^{* 12}}{z^{* 11}}-\frac{\sigma^{* 6}}{z^{* 5}}\right) \\
& -\lambda\left[\left(\frac{11}{2 z^{*}}+g^{*}\right) \alpha-\left(\frac{5}{2 z^{*}}+g^{*}\right) \beta\right] \\
\cdot & {\left[2 \cos \left(\frac{2 \pi}{\sqrt{3}} y^{*}\right) \cos \left(2 \pi x^{*}\right)+\cos \left(\frac{4 \pi}{\sqrt{3}} y^{*}\right)\right] . }
\end{aligned}
$$

In the same way, the lateral interaction force between the atom/ion and graphene in the polar coordinate system can be derived,

$$
\begin{aligned}
F_{t}^{*}= & -2 \pi \lambda(\alpha-\beta) \\
& \cdot\left[2 \cos \left(\frac{2 \pi}{\sqrt{3}} \rho^{*} \sin \theta\right) \sin \left(2 \pi \rho^{*} \cos \theta\right)\right],
\end{aligned}
$$

where $\rho^{*}$ and $\theta$ are dimensionless polar coordinates and $\rho^{*}$ coincides with the $x^{*}$-axis when the angle $\theta=0$.

\section{Results and Discussions}

Equation (13) describes the interaction potential between a single adsorption atom/ion and graphene via the L-J potential and the first-order approximation of Fourier expansion. Obviously, it is dependent on the position of the adsorption atom/ion on graphene and the parameters of L-J potential which is varied for different atom/ion. In this work, several kinds of metal atoms and ions are selected, including $\mathrm{Au}, \mathrm{Pt}$, $\mathrm{Mn}^{2+}, \mathrm{Na}^{1+}$, and $\mathrm{Li}^{1+}$. If the L-J potential parameters for these atoms/ions are specified [30-32], the interaction potential of these atoms/ions can be obtained in terms of the position vector above the graphene surface.

For a lithium-ion, the L-J potential parameters can be specified as $\sigma^{*}=1.005$ and $z^{*}=0.983$. The variations of interaction potential between the lithium-ion and graphene are illustrated in Figure 2 with respect to the positions in the $x$ - and $y$-directions, respectively. It can be seen from Figure 2 that the potential curves are periodic in both directions, and there are three periodic equilibrium positions, corresponding to three particular adsorption sites of lithiumion on graphene surface. They are hollow (on top of a hexagon, $\mathrm{H}-$ ), top (on top of a $\mathrm{C}-\mathrm{C}$ bond, $\mathrm{T}-$ ), and bridge (on top of a carbon atom, B-) sites (as shown in Figure 1(b)), respectively. At three adsorption sites, the equilibrium height and interaction potential at the equilibrium which can be called binding energy are different. Figure 3 shows the variations of interaction potential of the lithium-ion at three 


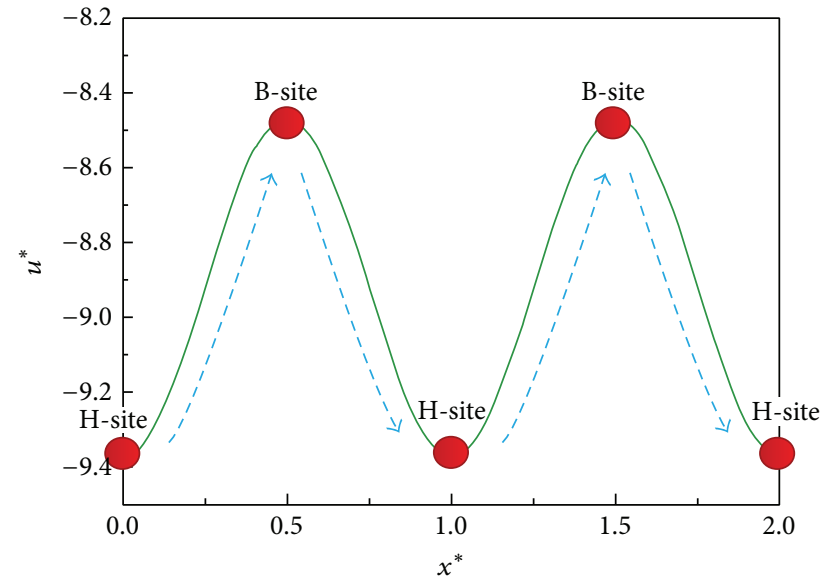

(a)

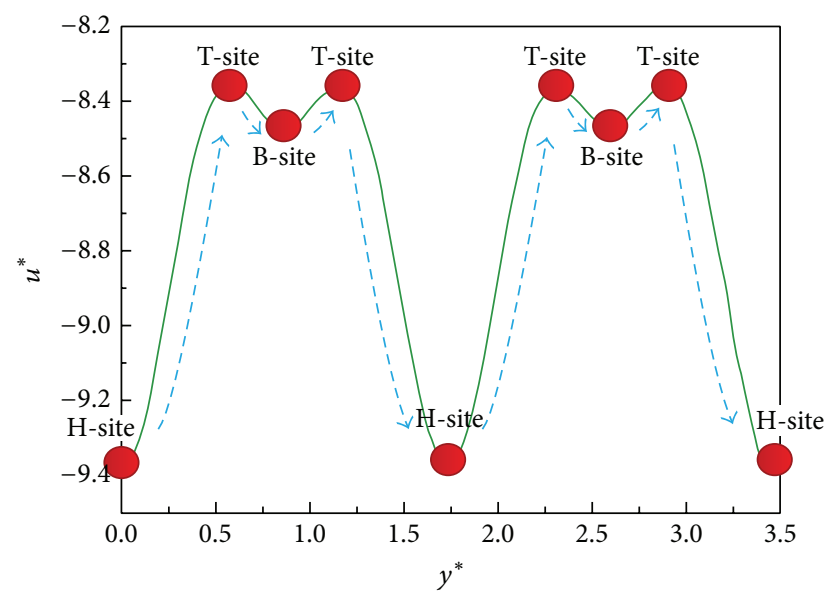

(b)

FIGURE 2: The variations of interaction potential between the lithium-ion and graphene in the (a) $x$-direction and (b) $y$-direction, respectively.

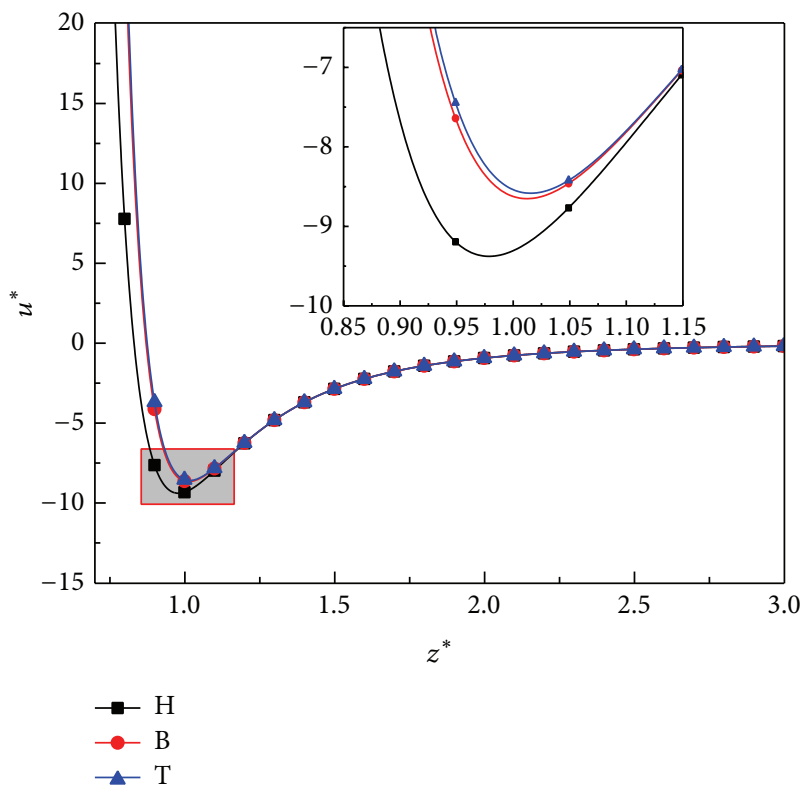

FIgURE 3: The variations of interaction potential of the lithium-ion at three adsorption sites along the $z$-direction.

adsorption sites along the $z$-direction. Although the variation trends are almost identical for the three sites, the equilibrium height and binding energy are different. The equilibrium height and binding energy at the $\mathrm{H}$-site are the lowest, followed by those at the B-site, and those at the T-site are the highest.

Furthermore, via the equilibrium equations of lithiumion in the $z$-direction, $F_{z}^{*}\left(x^{*}, y^{*}\right)=0$, the equilibrium height $z_{0}$ at three adsorption sites can be calculated, which is $0.241717 \mathrm{~nm}, 0.248800 \mathrm{~nm}$, and $0.249502 \mathrm{~nm}$, respectively. Compared with the results in the literature [30], there is a good agreement. The equilibrium height is very important for the design of lithium-ion battery based on graphene electrode materials, which can be used in predicting the minimum interlayer spacing $2 z_{0}$ of two parallel graphene sheets so that the embedded $\mathrm{Li}^{1+}$ undergoes no net force. According to our theoretical results, the minimum interlayer spacing for $\mathrm{Li}^{1+}$ should be approximately $0.48-0.50 \mathrm{~nm}$. With the equilibrium heights at three adsorption sites, the binding energy $u_{0}$ of lithium-ion can also be calculated. The minimum value of binding energy is $-0.04059 \mathrm{eV}$ at the $\mathrm{H}$-site, subsequently $-0.03746 \mathrm{eV}$ at the $\mathrm{B}$-site, and the maximum value is $-0.03717 \mathrm{eV}$ at the T-site. Thus, $\mathrm{H}$-site is the most stable adsorption site, and T-site is the most unstable site.

Similarly, when the L-J potential parameters of other atoms/ions are given, the variations of interaction potential of these atoms/ions can be illustrated with respect to the positions in the $x$-, $y$-, and $z$-directions, respectively (as shown in Figure 4). It can be found that the variation trends of interaction potential are almost the same in three directions for these atoms/ions. Three adsorption sites ( $\mathrm{H}_{-}, \mathrm{B}-$, and T-site) exist for every atom/ion on graphene surface. The equilibrium height and binding energy of these atoms/ions can be calculated and listed in Tables 1 and 2. Similar conclusions can be drawn that the order from low to high for both equilibrium height and binding energy is $\mathrm{H}$-site, $\mathrm{B}$-site, and $\mathrm{T}$-site and $\mathrm{H}$-site is the most stable adsorption site. In addition, the equilibrium height and binding energy of every atom/ion are different. Obviously, $\mathrm{Pt}$ atom adsorbed at the $\mathrm{H}$ site is the most stable among the atoms/ions selected in the work.

As the discussions above, the interaction potentials between adsorption atoms/ions and graphene are not constant but dependent on their positions on graphene surface. For different adsorptions sites, the energy for desorption of these atoms/ions is different. When an atom/ion migrates from an adsorption site to another, it must get over an energy barrier. The energy or force to drive the atom/ion is defined as 


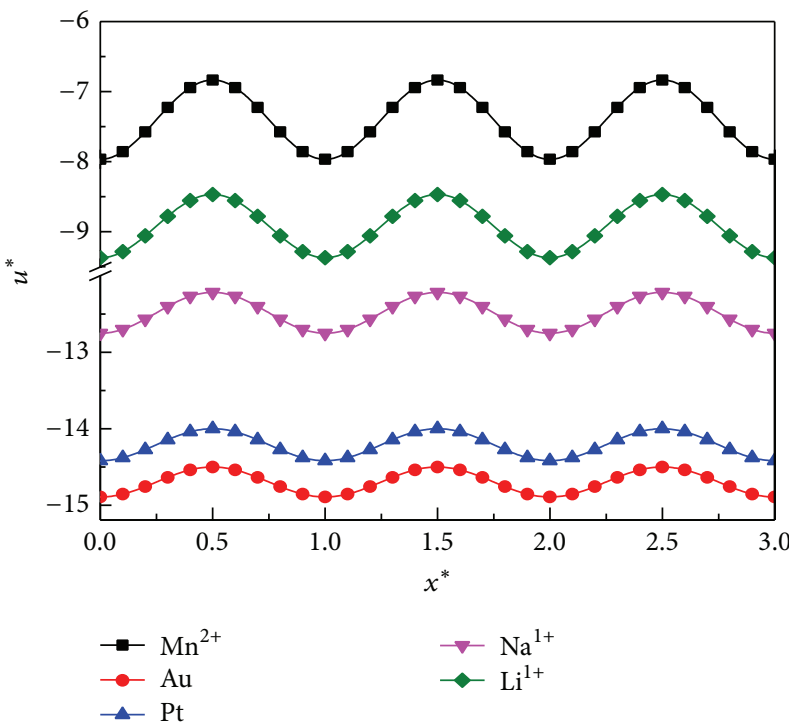

(a)

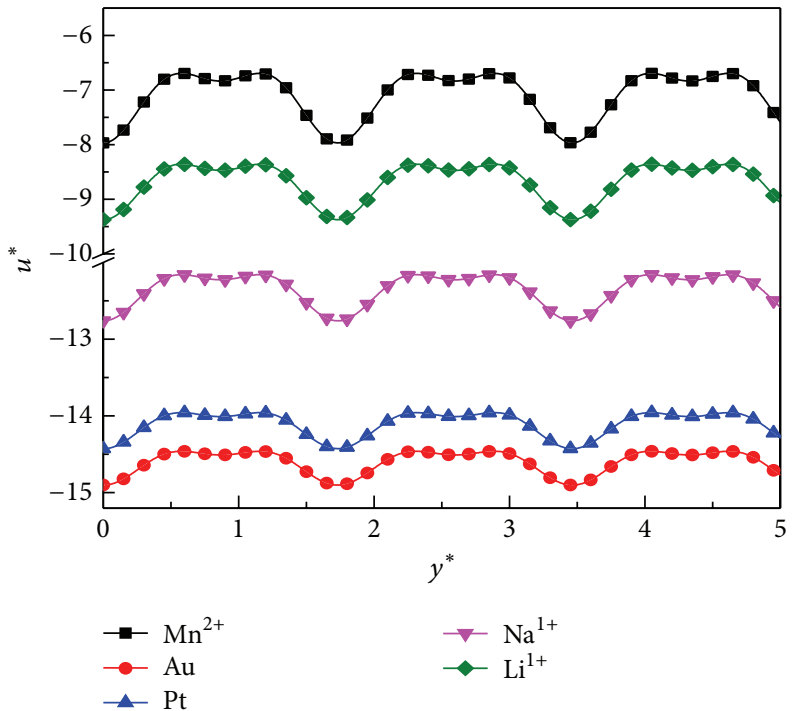

(b)

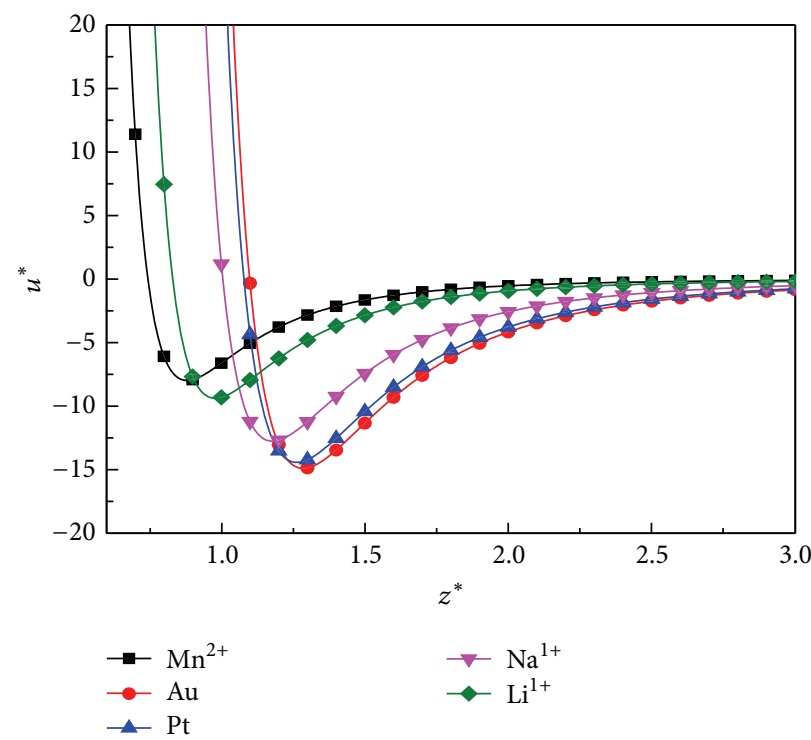

(c)

Figure 4: The variations of interaction potential of several kinds of metal atoms/ions along the (a) $x$-direction, (b) $y$-direction, and (c) $z$-direction, respectively.

TABLE 1: Equilibrium height $z_{0}$ of atoms/ions on the three particular adsorption sites.

\begin{tabular}{|c|c|c|c|c|c|c|}
\hline \multirow{2}{*}{ Equilibrium height (unit: nm) } & \multirow{2}{*}{ Position } & \multicolumn{5}{|c|}{ Atom/ion } \\
\hline & & $\mathrm{Mn}^{2+}$ & $\mathrm{Au}$ & $\mathrm{Pt}$ & $\mathrm{Na}^{1+}$ & $\mathrm{Li}^{1+}$ \\
\hline \multirow{3}{*}{$z_{0}$} & $\mathrm{H}$ & 0.218047 & 0.316354 & 0.310880 & 0.290234 & 0.241717 \\
\hline & B & 0.226746 & 0.319419 & 0.314168 & 0.294467 & 0.248800 \\
\hline & $\mathrm{T}$ & 0.227549 & 0.319769 & 0.314540 & 0.294932 & 0.249502 \\
\hline
\end{tabular}

TABLE 2: Binding energy $u_{0}$ of atoms/ions on the three particular adsorption sites.

\begin{tabular}{|c|c|c|c|c|c|c|}
\hline \multirow{2}{*}{ Binding energy (unit: eV) } & \multirow{2}{*}{ Position } & \multicolumn{5}{|c|}{ Atom/ion } \\
\hline & & $\mathrm{Mn}^{2+}$ & $\mathrm{Au}$ & $\mathrm{Pt}$ & $\mathrm{Na}^{1+}$ & $\mathrm{Li}^{1+}$ \\
\hline \multirow{3}{*}{$u_{0}$} & $\mathrm{H}$ & -0.07915 & -0.03127 & -0.10358 & -0.02936 & -0.04059 \\
\hline & $\mathrm{B}$ & -0.07084 & -0.03051 & -0.10084 & -0.02826 & -0.03746 \\
\hline & $\mathrm{T}$ & -0.07013 & -0.03043 & -0.10053 & -0.02814 & -0.03717 \\
\hline
\end{tabular}




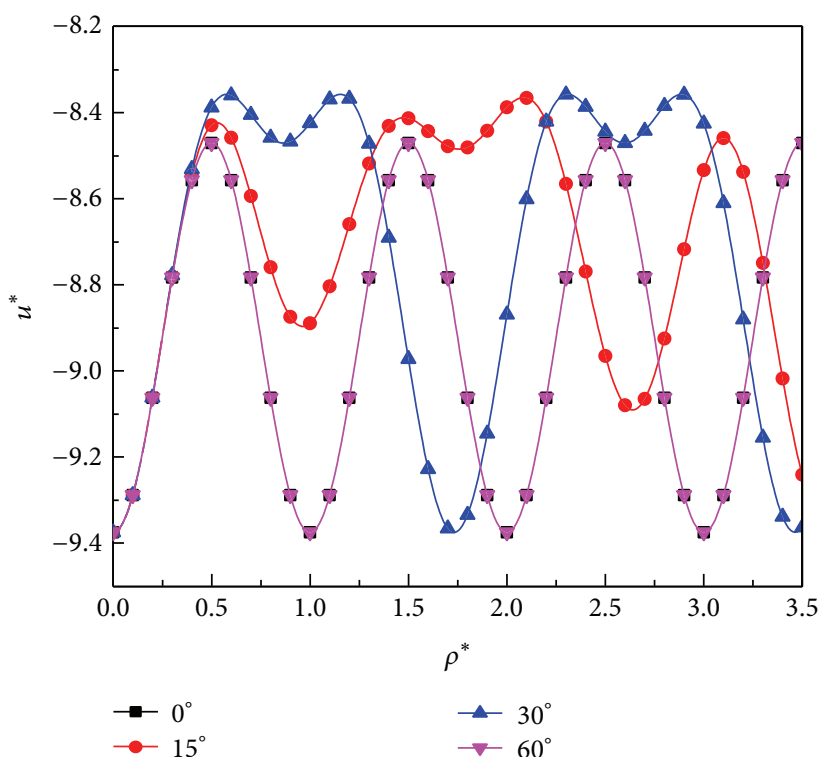

(a)

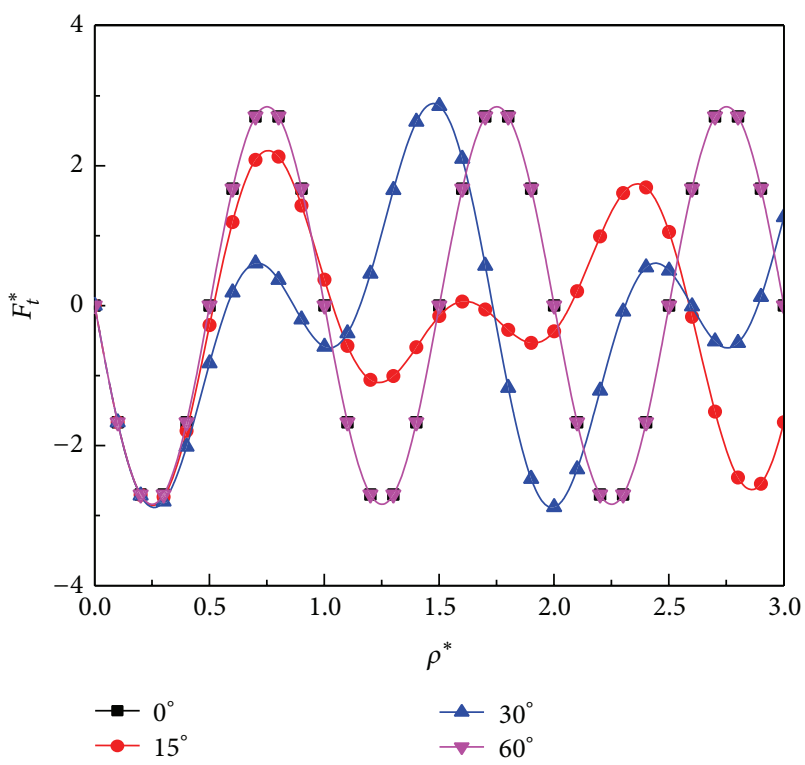

(b)

FIGURE 5: The variations of (a) interaction potential and (b) interaction force between a lithium-ion and graphene for different diffusion directions, $0^{\circ}, 15^{\circ}, 30^{\circ}$, and $60^{\circ}$ angles from the $x$-axis.

diffusion energy or diffusion force. Obviously, the diffusion energy and diffusion force are dependent on the diffusion path.

For a lithium-ion, the periodic interaction potentials along the $x$-and $y$-directions are illustrated in Figure 2. The period is $a$ in the $x$-direction and $\sqrt{3} a$ in the $y$-direction. If its initial adsorption site is assumed to be $\mathrm{H}$-site, in order to migrate along the $x$-direction, a lithium-ion must get over an energy barrier between $\mathrm{H}$-site and $\mathrm{B}$-site, and the minimum diffusion energy is $0.00313 \mathrm{eV}$. In the same way, in order to migrate along the $y$-direction, a lithium-ion must get over an energy barrier between $\mathrm{H}$-site and $\mathrm{T}$-site, and the minimum diffusion energy is $0.00342 \mathrm{eV}$. Similar results can also be obtained for other atoms/ions according to the periodic interaction potentials illustrated in Figure 4 and the binding energies listed in Table 2 .

The initial adsorption site is still assumed to be $\mathrm{H}$-site, and a lithium-ion is assumed to migrate along different directions, such as $0^{\circ}, 15^{\circ}, 30^{\circ}$, and $60^{\circ}$ angles from the $x$-axis. The dimensionless interaction potential and interaction force between the lithium-ion and graphene for different diffusion directions are illustrated in Figure 5. It can be seen from these figures that the periods of interaction potential and interaction force are varied for different diffusion directions, and the minimum diffusion energy and diffusion force are also varied; that is, the diffusion of atoms/ions on graphene surface is path-dependent. Simultaneously, it can be found that along the same lattice orientation, such as $0^{\circ}$ and $60^{\circ}$ angles, the variations of interaction potential and interaction force are nearly the same. These phenomena have been verified by the atomic scale friction experiments of AFM tip on graphene surface [33-35].
Furthermore, the diffusion from different initial positions along $x$ - and $y$-directions is investigated. The dimensionless interaction potential and interaction force of a lithiumion from different initial sites are illustrated along $x$ - and $y$-directions in Figures 6 and 7. In two directions, the periods of interaction potential and interaction force have path-independent period, $a$ and $\sqrt{3} a$, respectively. However, for different diffusion paths along $x$ - or $y$-direction, their amplitudes are varied; that is, the minimum diffusion energy and diffusion force are varied.

\section{Conclusions}

By performing theoretical analyses of interaction potential and interaction force for a single atom/ion on graphene surface based on an analytical model, the mechanisms of adsorption and diffusion are revealed. The equilibrium height and binding energy on three particular adsorption sites are calculated, and the adsorption stability is discussed. The order from low to high for both equilibrium height and binding energy is $\mathrm{H}$-site, $\mathrm{B}$-site, and $\mathrm{T}$-site, and $\mathrm{H}$ site is the most stable adsorption site. The variations of interaction potential and interaction force of lithium-ion diffusing along some specific directions on graphene surface are investigated, and it can be concluded that the diffusion of atoms/ions on graphene surface is path-dependent. The minimum diffusion energy and diffusion force along different diffusing path can be calculated by the analytical model.

The studies on the path-dependent diffusion of atoms/ions on graphene surface are beneficial to improve 


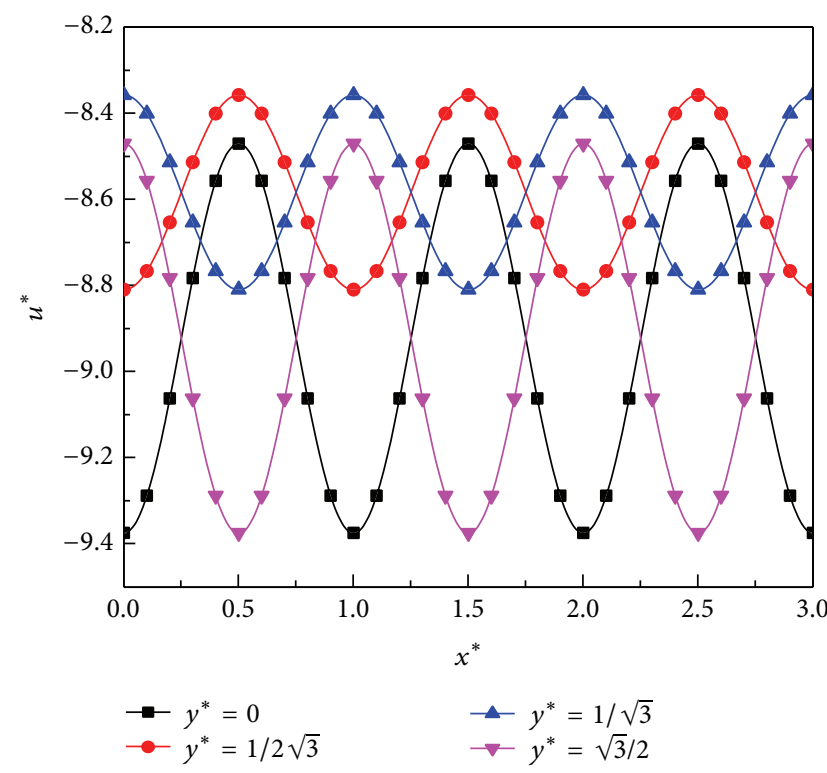

(a)

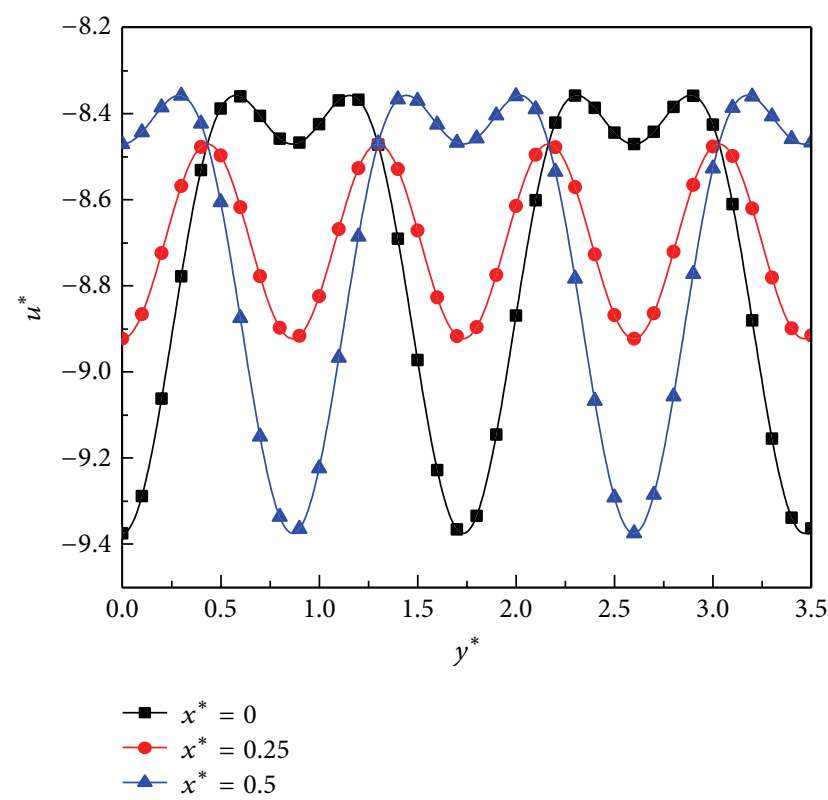

(b)

FIGURE 6: The variations of interaction potential between a lithium-ion and graphene from different initial sites along (a) $x$-direction and (b) $y$-direction.

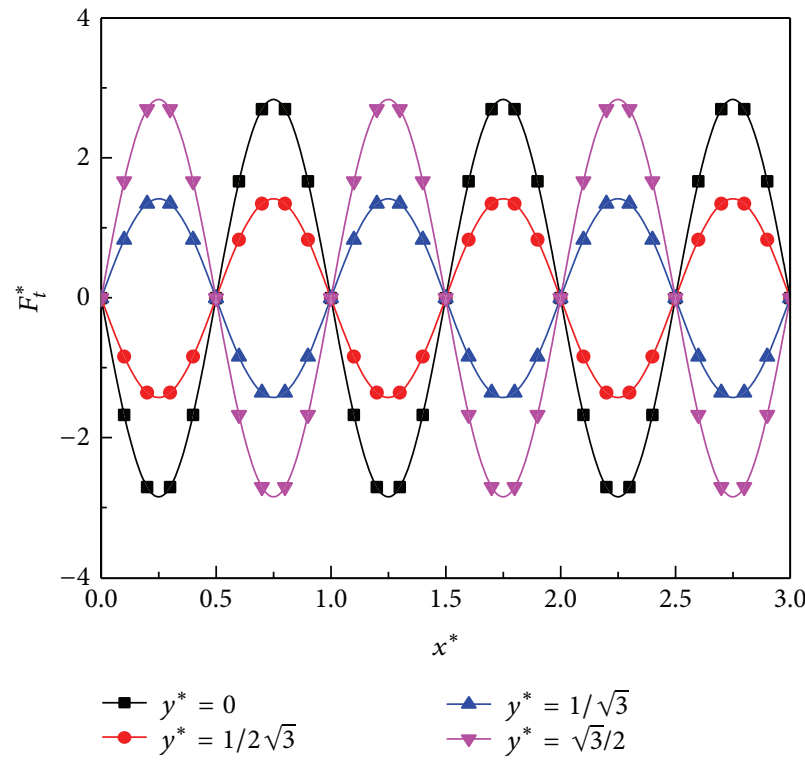

(a)

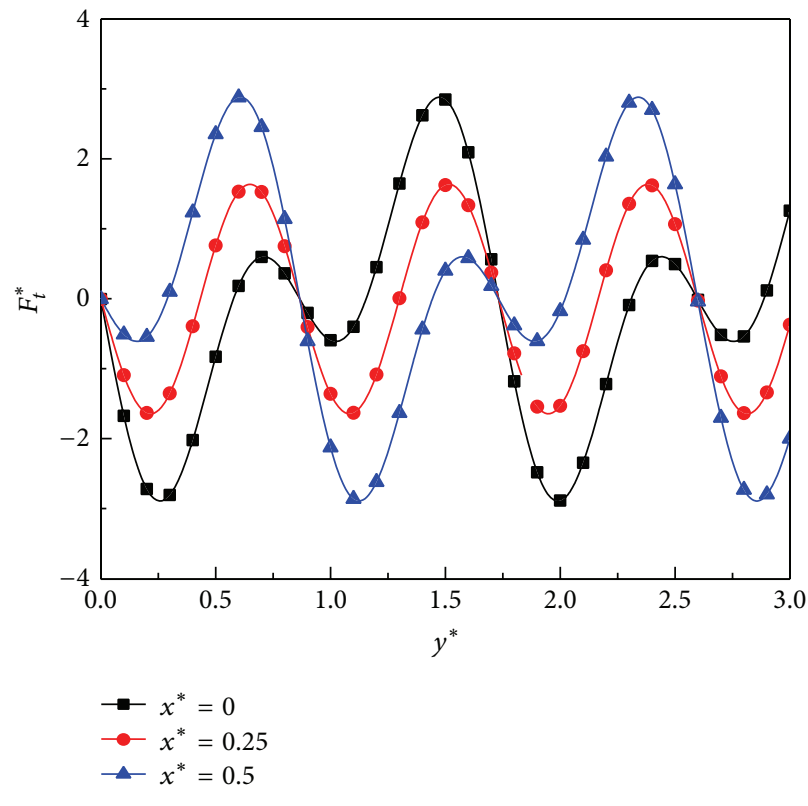

(b)

FIGURE 7: The variations of interaction force between a lithium-ion and graphene from different initial sites along (a) $x$-direction and (b) $y$-direction.

the efficiency of drug delivery based on graphene, highperformance rechargeable lithium-ion batteries, and lithium storage in carbon materials, which can be further applied for the interaction between nanoparticles or AFM tip and graphene surface. Of course, there is a little inadequacy in present studies. For example, the graphene is assumed to be infinite, perfect, and flat; moreover, the influence of temperature is not taken into account. In fact, edge effect, temperature, defect and corrugation of graphene, and so forth can affect the adsorption and diffusion of atom/ion $[30,36-40]$. Therefore, these factors will be considered in our future works. 


\section{Conflict of Interests}

The authors declare that there is no conflict of interests regarding the publication of this paper.

\section{Acknowledgments}

The work is supported by the National Natural Science Foundation of China (Grants nos. 11372216, 11372217, and 11502167) and the National Basic Research Program of China (973 Program, Grant no. 2012CB937500).

\section{References}

[1] L. Huang, Z. P. Wang, J. K. Zhang et al., "Fully printed, rapidresponse sensors based on chemically modified graphene for detecting $\mathrm{NO}_{2}$ at room temperature," ACS Applied Materials and Interfaces, vol. 6, no. 10, pp. 7426-7433, 2014.

[2] J. Ma, W. Jin, H. L. Ho, and J. Y. Dai, "High-sensitivity fiber-tip pressure sensor with graphene diaphragm," Optics Letters, vol. 37, no. 13, pp. 2493-2495, 2012.

[3] J. H. Li, J. L. Liu, G. R. Tan et al., "High-sensitivity paracetamol sensor based on Pd/graphene oxide nanocomposite as an enhanced electrochemical sensing platform," Biosensors and Bioelectronics, vol. 54, pp. 468-475, 2014.

[4] K. Park, "Controlled drug delivery systems: past forward and future back," Journal of Controlled Release, vol. 190, pp. 3-8, 2014.

[5] Y. Zhang, H. F. Chan, and K. W. Leong, "Advanced materials and processing for drug delivery: the past and the future," Advanced Drug Delivery Reviews, vol. 65, no. 1, pp. 104-120, 2013.

[6] H. C. Zhang, G. Grüner, and Y. L. Zhao, "Recent advancements of graphene in biomedicine," Journal of Materials Chemistry B, vol. 1, no. 20, pp. 2542-2567, 2013.

[7] J. Q. Liu, L. Cui, and D. Losic, "Graphene and graphene oxide as new nanocarriers for drug delivery applications," Acta Biomaterialia, vol. 9, no. 12, pp. 9243-9257, 2013.

[8] J. Hassoun, F. Bonaccorso, M. Agostini et al., "An advanced lithium-ion battery based on a graphene anode and a lithium iron phosphate cathode," Nano Letters, vol. 14, no. 8, pp. 49014906, 2014.

[9] Z. Ji, F. F. Contreras-Torres, A. F. Jalbout, and A. RamírezTreviño, "Surface diffusion and coverage effect of $\mathrm{Li}$ atom on graphene as studied by several density functional theory methods," Applied Surface Science, vol. 285, part B, pp. 846-852, 2013.

[10] A. Buldum and G. Tetiker, "First-principles study of graphenelithium structures for battery applications," Journal of Applied Physics, vol. 113, no. 15, Article ID 154312, 2013.

[11] G. Kucinskis, G. Bajars, and J. Kleperis, "Graphene in lithium ion battery cathode materials: a review," Journal of Power Sources, vol. 240, pp. 66-79, 2013.

[12] E. J. Yoo, J. Kim, E. Hosono, H.-S. Zhou, T. Kudo, and I. Honma, "Large reversible Li storage of graphene nanosheet families for use in rechargeable lithium ion batteries," Nano Letters, vol. 8, no. 8, pp. 2277-2282, 2008.

[13] H. Yildirim, A. Kinaci, Z.-J. Zhao, M. K. Y. Chan, and J. P. Greeley, "First-principles analysis of defect-mediated Li adsorption on graphene," ACS Applied Materials and Interfaces, vol. 6, no. 23, pp. 21141-21150, 2014.
[14] J. J. Zhou, W. W. Zhou, C. M. Guan et al., "First-principles study of lithium intercalated bilayer graphene," Science China: Physics, Mechanics and Astronomy, vol. 55, no. 8, pp. 1376-1382, 2012.

[15] D. Krepel and O. Hod, "Lithium adsorption on armchair graphene nanoribbons," Surface Science, vol. 605, no. 17-18, pp. 1633-1642, 2011.

[16] M. Amft, S. Lebègue, O. Eriksson, and N. V. Skorodumova, "Adsorption of $\mathrm{Cu}, \mathrm{Ag}$, and $\mathrm{Au}$ atoms on graphene including van der Waals interactions," Journal of Physics Condensed Matter, vol. 23, Article ID 395001, 2011.

[17] L. Xian and M. Y. Chou, "Diffusion of Si and C atoms on and between graphene layers," Journal of Physics D: Applied Physics, vol. 45, no. 45, Article ID 455309, 2012.

[18] Y. Xia, Z. Li, and H. J. Kreuzer, "Adsorption, diffusion and desorption of hydrogen on graphene," Surface Science, vol. 605, no. 21-22, pp. L70-L73, 2011.

[19] H. Tachikawa, "A direct molecular orbital-molecular dynamics study on the diffusion of the $\mathrm{Li}$ ion on a fluorinated graphene surface," Journal of Physical Chemistry C, vol. 112, no. 27, pp. 10193-10199, 2008.

[20] H. Tachikawa, T. Iyama, and H. Kawabata, "MD simulation of the interaction of magnesium with graphene," Thin Solid Films, vol. 518, no. 2, pp. 877-879, 2009.

[21] X. B. Zhao and P. Liu, "Biocompatible graphene oxide as a folate receptor-targeting drug delivery system for the controlled release of anti-cancer drugs," RSC Advances, vol. 4, no. 46, pp. 24232-24239, 2014.

[22] J. Rodríguez-López, N. L. Ritzert, J. A. Mann, C. Tan, W. R. Dichtel, and H. D. Abruña, "Quantification of the surface diffusion of tripodal binding motifs on graphene using scanning electrochemical microscopy," Journal of the American Chemical Society, vol. 134, no. 14, pp. 6224-6236, 2012.

[23] W. A. Steele, "The physical interaction of gases with crystalline solids: I. Gas-solid energies and properties of isolated adsorbed atoms," Surface Science, vol. 36, no. 1, pp. 317-352, 1973.

[24] W. A. Steele, "The physical interaction of gases with crystalline solids II. Two-dimensional second and third virial coefficients," Surface Science, vol. 39, no. 1, pp. 149-175, 1973.

[25] J.-A. Ruan and B. Bhushan, "Atomic-scale and microscale friction studies of graphite and diamond using friction force microscopy," Journal of Applied Physics, vol. 76, no. 9, pp. 5022$5035,1994$.

[26] M. Chi and Y.-P. Zhao, "Adsorption of formaldehyde molecule on the intrinsic and Al-doped graphene: a first principle study," Computational Materials Science, vol. 46, no. 4, pp. 1085-1090, 2009.

[27] M. Chi and Y.-P. Zhao, "First principle study of the interaction and charge transfer between graphene and organic molecules," Computational Materials Science, vol. 56, pp. 79-84, 2012.

[28] D. Qian, G. J. Wagner, W. K. Liu, M. Yu, and R. S. Ruoff, "Mechanics of carbon nanotubes," Applied Mechanics Reviews, vol. 55, no. 6, pp. 495-533, 2002.

[29] Y. Wang, D. Tománek, and G. F. Bertsch, "Stiffness of a solid composed of C60 clusters," Physical Review B, vol. 44, no. 12, pp. 6562-6565, 1991.

[30] Y. Chan and J. M. Hill, "Modelling interaction of atoms and ions with graphene," Micro and Nano Letters, vol. 5, no. 5, pp. 247250, 2010.

[31] B. J. Cox, N. Thamwattana, and J. M. Hill, "Mechanics of atoms and fullerenes in single-walled carbon nanotubes. I. Acceptance and suction energies," Proceedings of the Royal Society of London A, vol. 463, pp. 461-476, 2007. 
[32] J. M. Hill, N. Thamwattana, and B. J. Cox, "Mechanics of atoms and fullerenes in single-walled carbon nanotubes. II. Oscillatory behavior," Proceedings of the Royal Society of London A, vol. 463, no. 2078, pp. 477-494, 2007.

[33] O. Zwörner, H. Hölscher, U. D. Schwarz, and R. Wiesendanger, "The velocity dependence of frictional forces in point-contact friction," Applied Physics A: Materials Science and Processing, vol. 66, no. 1, pp. S263-S267, 1998.

[34] H. Hölscher, U. D. Schwarz, O. Zwörner, and R. Wiesendanger, "Consequences of the stick-slip movement for the scanning force microscopy imaging of graphite," Physical Review B, vol. 57, no. 4, pp. 2477-2481, 1998.

[35] Y. Zhang, L. Q. Liu, N. Xi, Y. C. Wang, Z. L. Dong, and U. C. Wejinya, "Friction anisotropy dependence on lattice orientation of graphene," Science China: Physics, Mechanics and Astronomy, vol. 57, no. 4, pp. 663-667, 2014.

[36] C. Uthaisar and V. Barone, "Edge effects on the characteristics of Li diffusion in graphene," Nano Letters, vol. 10, no. 8, pp. 28382842, 2010.

[37] L. Chen, H. Hu, Y. Ouyang, H. Z. Pan, Y. Y. Sun, and F. Liu, "Atomic chemisorption on graphene with Stone-Thrower-Wales defects," Carbon, vol. 49, no. 10, pp. 3356-3361, 2011.

[38] D. Datta, J. W. Li, N. Koratkar, and V. B. Shenoy, "Enhanced lithiation in defective graphene," Carbon, vol. 80, no. 1, pp. 305310, 2014.

[39] Z. H. Aitken and R. Huang, "Effects of mismatch strain and substrate surface corrugation on morphology of supported monolayer graphene," Journal of Applied Physics, vol. 107, no. 12, Article ID 123531, 2010.

[40] W. Gao and R. Huang, "Effect of surface roughness on adhesion of graphene membranes," Journal of Physics D: Applied Physics, vol. 44, no. 45, Article ID 452001, 2011. 

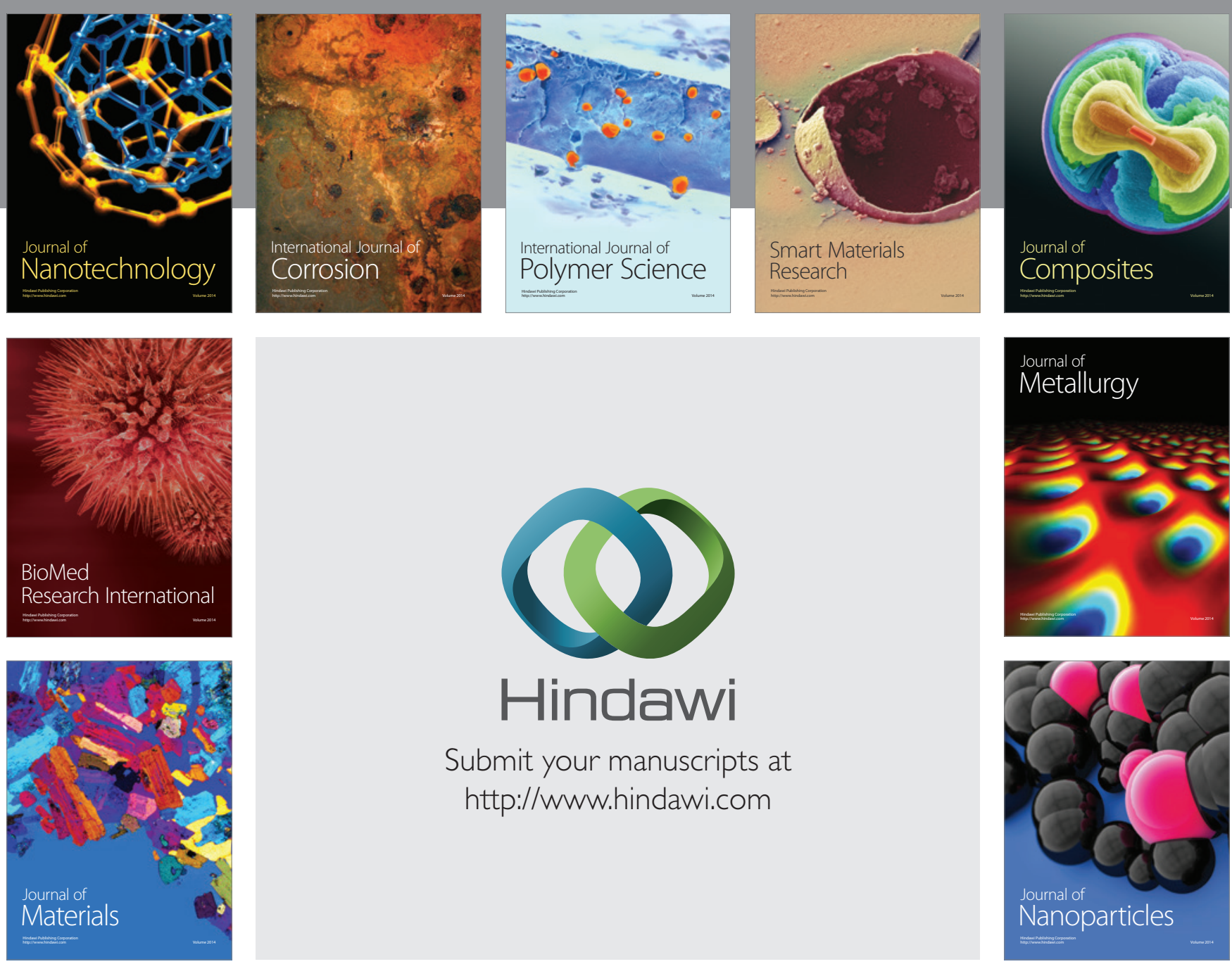

Submit your manuscripts at http://www.hindawi.com
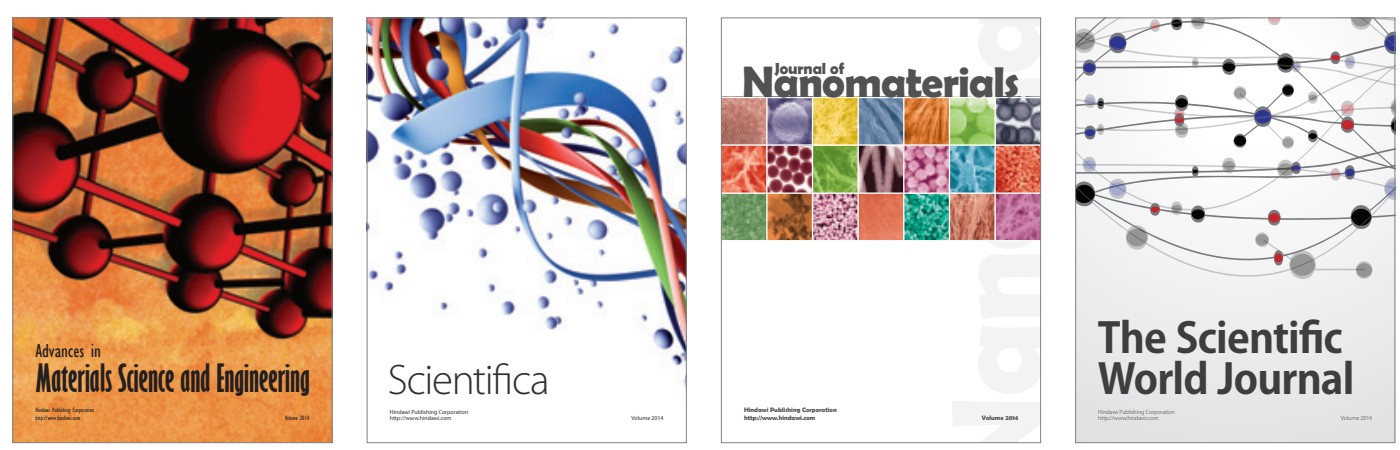

\section{The Scientific World Journal}
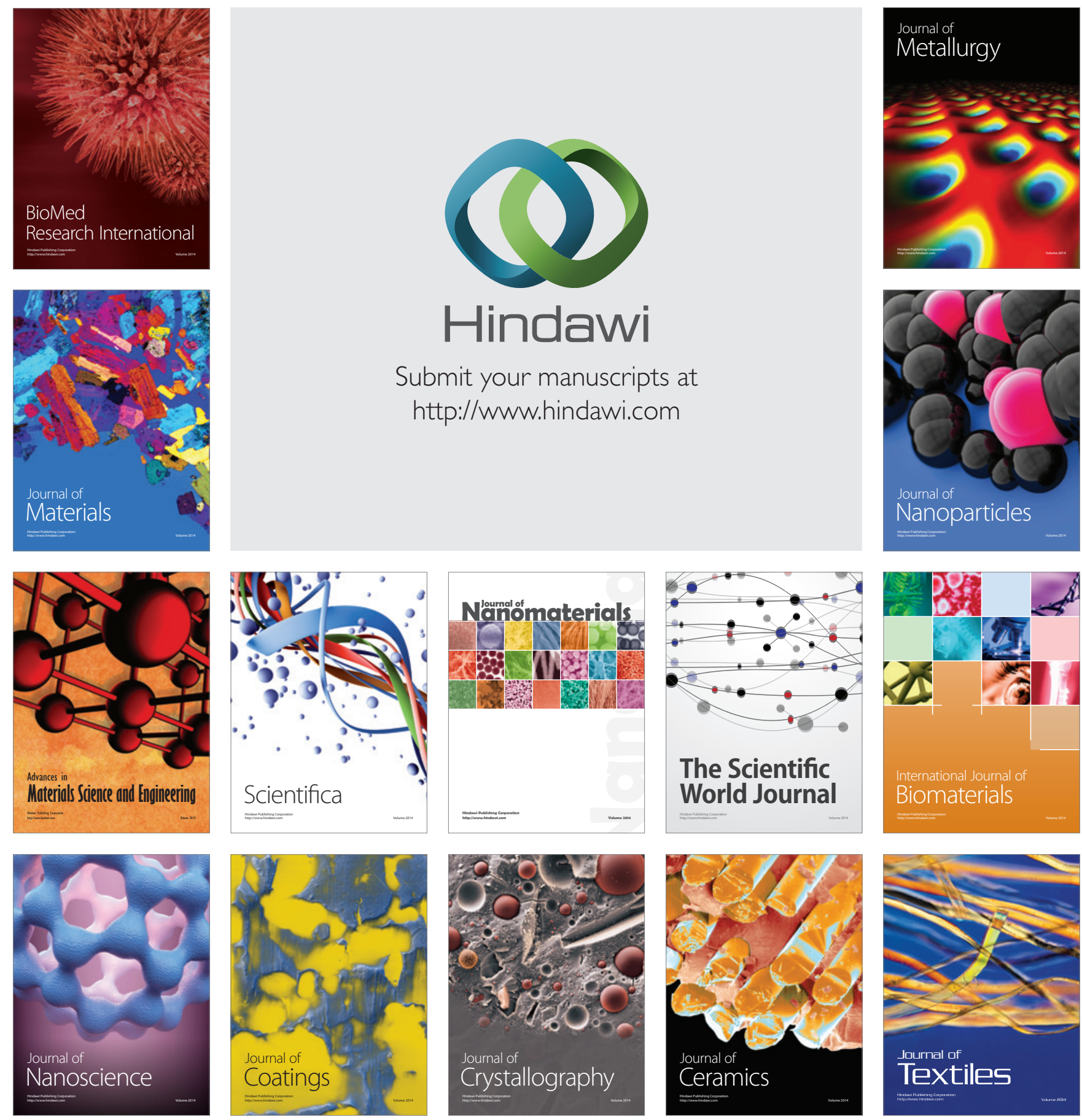